\title{
Metastatic trophoblastic disease masquerading as pulmonary embolism
}

A 51-year-old woman presented with a 2week history of dyspnoea, dry cough and fever which did not improve with a course of oral antibiotics. Her past medical history was significant for a termination of pregnancy more than 5 years previously and a total hysterectomy and bilateral salpingo-oophorectomy shortly thereafter for infarcted fibroids. Physical examination revealed decreased breath sounds over the left lower chest. The chest radiograph showed infiltrates in the left lower zone associated with a small pleural effusion. A CT scan of the thorax (fig 1A) revealed an intraluminal filling defect in the distal left pulmonary artery consistent with a diagnosis of pulmonary embolism for which anticoagulation was promptly started. Lower limb duplex ultrasound was negative for deep venous thrombosis and a CT scan of the abdomen and pelvis did not demonstrate any intraabdominal or intrapelvic masses. Despite adequate anticoagulation over a 3-month period, the patient failed to improve and had episodes of haemoptysis. A second CT scan of the thorax was performed which showed an increase in the size of the left pulmonary artery clot and new
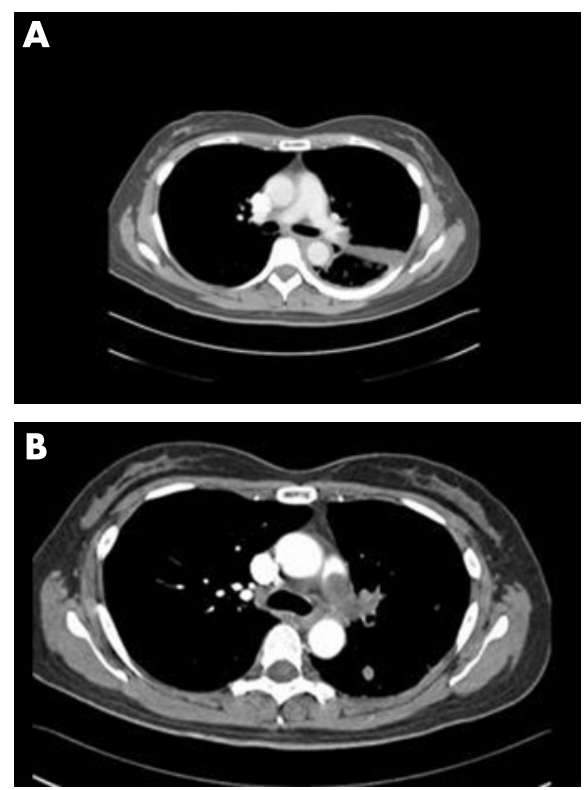

Figure 1 (A) Initial CT scan showing intraluminal filling defect and (B) CT scan 3 months later showing increase in size despite anticoagulation. pulmonary nodules in the left lung (fig 1B). Positron emission tomography combined with CT (PET-CT) showed a hypermetabolic lesion projected over the left pulmonary artery with a standardised uptake value of 8.2 as well as multiple pulmonary
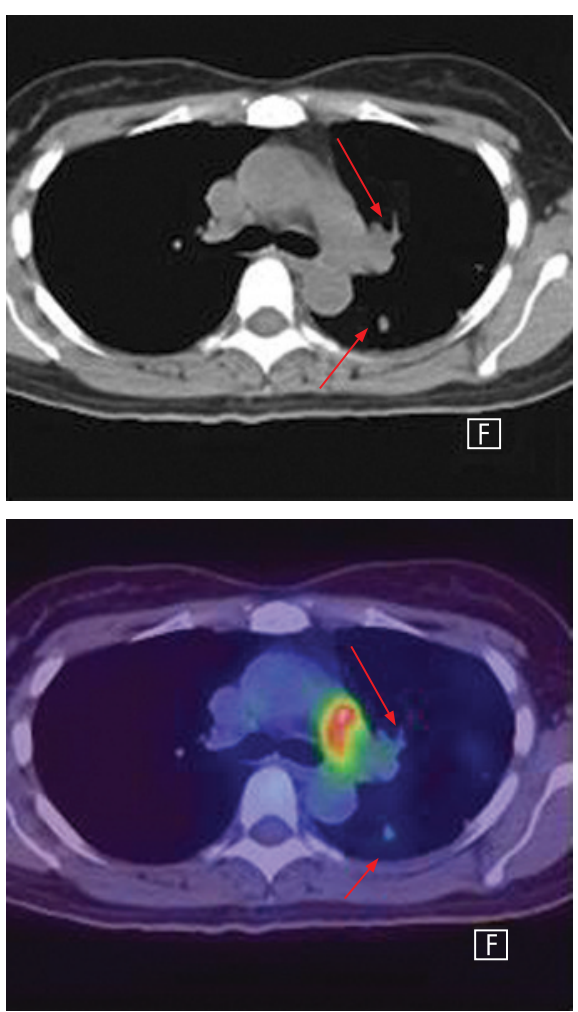

Figure 2 Positron emission tomography combined with CT scanning (PET-CT) showing a hypermetabolic lesion projected over the left pulmonary artery and two smaller lung nodules (red arrows). The reduced level of PET activity associated with the two left pulmonary nodules is probably secondary to their small sizes.

\section{Learning points}

- Pulmonary artery malignancy should be considered when symptoms persist or worsen in the presence of adequate anticoagulation therapy.

- Metastatic trophoblastic disease should be considered in women after hysterectomy who present with symptoms suggestive of pulmonary embolism.

- Positron emission tomography combined with CT scanning may be helpful to differentiate pulmonary artery neoplasms, both primary and metastatic, from organising embolus.

nodules of varying hypermetabolism and sizes (fig 2). Bronchoscopy revealed nothing unusual so a left lung wedge resection was performed via video-assisted thorascopic surgery which showed poorly differentiated metastatic choriocarcinoma. She was commenced on chemotherapy.

Primary and metastatic choriocarcinoma have been reported to mimic pulmonary embolism in women after pregnancy and after abortion. ${ }^{1-3}$ However, reports of trophoblastic disease occurring many years after hysterectomy are rare, as in this case, but should be considered in the differential diagnosis of an unresolving pulmonary embolism. PET-CT scanning may help to differentiate pulmonary artery neoplasms, both primary and metastatic, from organising embolus. ${ }^{4}$

\section{Ong, S Y Low, A Thirugnanam, A Loh, P Eng Singapore General Hospital, Singapore}

Correspondence to: Dr C Ong, Singapore Genera Hospital, Outram Road, Singapore 169608; catongwm@ yahoo.com

\section{Competing interests: None.}

Thorax 2008;63:1030. doi:10.1136/thx.2007.084533

\section{REFERENCES}

1. Brusselle G, Van Nooten G, Delrue L, et al. Cor pulmonale and respiratory failure in a young woman. Respiration 2005:72:549-51.

2. Watanabe S, Shimokawa S, Sakasegawa K, et al. Choriocarcinoma in the pulmonary artery treated with emergency pulmonary embolectomy. Chest 2002;121:654-6.

3. Kumar J, llancheran A, Ratnam SS. Pulmonary metastases in gestational trophoblastic disease: a review of 97 cases. Br J Obstet Gynaecol 1988:95:70-4.

4. Kim JH, Gutierrez FR, Lee EY, et al. Primary leiomyosarcoma of the pulmonary artery: a diagnostic dilemma. J Clin Imaging 2003:27:206-11.

5. Lai $\mathbf{P}$, Bomanji JB, Mahmood S, et al. Detection of tumour thrombus by 18F-FDG-PET/CT imaging. Eur J Cancer Prev 2007;16:90-4. 